\title{
LÍNEA DE INTELIGENCIA ARTIFICIAL Y PROCESOS DE RAZONAMIENTO
}

\author{
Luis Facundo Maldonado Granados*, PhD
}

\begin{abstract}
This paper is an introduction for the fine of research on Artificial Intelligence and qualitative reasoning in the framework of the Doctor in Science Teaching Area of the Interinstitutional Program of Doctor in Education. Main features of the field are introduced by means of a brief review of trends and issues in the Artificial Intelligence in general, and particularly in education. There is a short description of academic initiatives and developments in the universities which support the program. There are statements about the theoretical approach, methods of research, technological fundamentals and pedagogical orientation. Curriculum activities and bibliographical references provide additional information on the focus of this fine of research..
\end{abstract}

\section{Justificación}

\section{Antecedentes conceptuales}

Filosóficamente la Inteligencia Artificial fue viable en la medida en que se superó la concepción del dualismo mente-cuerpo. Algunas posiciones teóricas reflejan este largo proceso. Una primera tesis fuerte sostiene que el pensamiento es una forma de actividad física y, por tanto, puede ser llevada a cabo por sistemas también físicos tales como el cerebro o un tipo de máquina que lo emule. Otra demuestra que el proceso cognoscitivo se puede caracterizar de manera apropiada a través de alguna forma de matemática. al decir de Hume, "el conocimiento es cálculo" (Lugger and Sutbblefield, 1993: pg. 6). Babage sostuvo que cualquier cosa que se pueda representar en un sistema de símbolos puede ser procesada por un cómputador ${ }^{1}$. Finalmente, surge la concepción de que el cerebro es un sistema de procesamiento de información: el computador es una metáfora del mismo.

\section{Antecedentes metodológicos}

Esta evolución del pensamiento abrió camino a y fue acompañada por el estudio de la lógica como formalización de la actividad mental. Los aportes de Euler (1735), relacionados con el análisis de sistemas conectados, dan origen a la teoría de grafos posteriormente usada en la representación de espacios de problema. Boole (1847 y 1954) investiga las operaciones del pensamiento y se propone darles expresión por medio de un lenguaje de símbolos, establece los fundamentos de la lógica proposicional. Este enfoque -de representar por medio de símbolos los procesos de la mente-se ubica posteriormente en el corazón de la Inteligencia Artificial. Frege $(1879,1884)$ establece las bases del cálculo de predicados de primer orden que posteriormente se convierte en lenguaje de programación a través de Prolog. Indudablemente la lógica dota a la Inteligencia Artificial

\footnotetext{
*Profesor Titular Universidad Pedagógica Nacional. Programa interinstitucional de Doctorado en Educación: Área Educación en Ciencias Naturales.

${ }^{1}$ Esta formulación es extraída en su sentido de las notas de Ada Augusta Countess Lovelace en relación con la maquina de Babage, en Shapiro (1987).
} 
de los mecanismos y métodos indispensables para una eficiente representación simbólica de los procesos de razonamiento.

Un aporte tanto teórico como metodológico previo a los desarrollos de la Inteligencia Artificial proviene del trabajo de Shannon y Weaver (1949) en el cual se formaliza el concepto de información y se desarrolla una metodología para su medición. Esta conceptualización en términos de variedad trasmitida entre sistemas abre paso a la especificación de mecanismos de procesamiento.

Von Newman diseña un modelo de procesamiento de información con entradas y salidas donde se separan las funciones de memoria de las de control y se caracteriza el procesador como mecanismo que opera por transición de estados (Fortier, 1986).

Turing (1936) desarrolla el concepto de máquina universal capaz de replicarse a sí misma. La teoría de autómatas finitos demuestra que cualquier cálculo, por complejo que sea, puede descomponerse en procesos elementales, que cualquier problema puede teóricamente solucionarse mediante una cadena de pasos discretos y que es posible diseñar una máquina capaz de resolver cualquier tipo de cálculo. Von Newman, basado en este principio, analizó las posibilidades de la reproducción biológica, en una excelente anticipación de lo que actualmente se denomina vida artificial (Singh, 1966).

\section{Trabajos pioneros}

La convergencia de estudios sobre la inteligencia y diseño de dispositivos programables -computadores- da lugar al surgimiento de una tendencia de investigación interesada en generar programas capaces de emular actividades propias del ser humano - en principio- y, en general, de los seres vivos. En el verano de 1956, en la realización de un seminario de investigadores ${ }^{2}$, se acuña la expresión Inteligencia Artificial para denominar este movimiento. Para esta época se tenían algunas realizaciones que se constituyeron en el punto de partida de la formación de un cuerpo de conocimiento especialmente promisorio.

Warren McCulloch y Walter Pitts (1943) desarrollaron un modelo de cómputo usando como referente la neurona, capaz de aprender de la experiencia y Marvin Minsky y Dean Edmons construyeron la primera computadora neuronal en 1951. Shannon (1950) y Turing (1953) desarrollaron los primeros programas de ajedrez. Arthur Samuel (1959) desarrolló un programa de computador para el juego de damas que aprendió a jugar hasta superar a su creador. Alíen Newell y Herbert Simon (1956) desarrollaron The Logic Theorist capaz de probar teoremas, demostrando las enormes posibilidades del computador para el procesamiento simbólico como superación del enfoque numérico.

La trascendencia de estas realizaciones muy posiblemente se debe a que estuvieron acompañadas de logros a nivel teórico y metodológico. La tríada de componentes: teoría, metodología y dispositivo acompañan los desarrollos de Inteligencia Artificial hasta el presente.

\footnotetext{
${ }^{2}$ El seminario celebrado en Darmouth. convocado por J. McCarthy. 


\section{Caracterización del campo de estudio}

Tanimoto (1987: 7) expresa que el propósito fundamental de la IA es mejorar la comprensión de los procesos de razonamiento, aprendizaje y percepción del ser humano. Los principales retos del desarrollo de este campo están constituidos por la representación de conocimiento, búsqueda en los procesos de solución de problemas, percepción e inferencia.

El conocimiento se halla disponible en la sociedad, en primera instancia, a través de las personas y -en segundo lugar- de dispositivos de almacenamiento como libros y otros. Son las personas quienes muestran su dinámica integral. Los investigadores, basados en su distribución, distinguen conocimiento de sentido común y conocimiento experto. Una tarea básica en IA es adquirirlo y representarlo en sus rasgos esenciales. En la práctica, este proceso produce conjuntos de proposiciones lógicas, reglas, procedimientos, heurísticas, estructuras, etc., resultados que dan lugar a considerar subcategorías como conocimiento esencial, de control, procedimental, heurístico y declarativo (Rich y Knight 1996). Una buena representación debe garantizar facilidad para su identificación, evaluación, actualización e incorporación en los procesos de razonamiento de sistemas artificiales.

En la medida en que se avanza en la IA, la práctica de usar metáforas como estrategia de estudio de los problemas se ha mostrado rica en consecuencias, debido tanto a la posibilidad de ir de lo más conocido a lo desconocido, como por su enfoque estructural y holistico (Maldonado y Vargas 1994). Un enfoque integral de los retos de la IA se muestra en la metáfora del agente inteligente. Russell y Norvig (1996: 33) expresan: "un agente es todo aquello que puede considerarse que percibe su ambiente mediante censores y que responde o actúa en tal ambiente por medio de efectores. Para ser inteligente requiere mostrar características de racionalidad y autonomía.

Si revisamos el planteamiento de Tanimoto (1987) que señala que el razonamiento, el aprendizaje y la percepción son los principales retos de la IA, el concepto de agente muestra una evolución en términos de integralidad. En efecto, éste incorpora de manera holistica estos tres componentes, pero, además, los proyecta a la dinámica de la acción y de la interacción con un ambiente.

Un agente es un ente situado en un entorno, en el cual desarrolla percepciones, base de su aprendizaje. Está informado en la medida en que percibe, sin que esto sea suficiente para su existencia como ente activo. En efecto, el agente ideal es racional. Esto significa que la información que obtiene alimenta procesos de razonamiento que conducen a decisiones, las cuales, a su vez son activadores de acciones. La acción, por su parte, compromete a los mecanismos efectores, a través de los cuales el agente modifica al mundo que lo rodea.

Esta descripción es suficiente para un agente activo, pero, no para un agente inteligente. La inteligencia exige la autonomía, la cual, requiere como condiciones de posibilidad la formulación de metas, un conocimiento incorporado a la estructura del agente, un continuo experiencial -secuencia de percepciones-, la identificación de alternativas de acción y la activación de un mecanismo decisor que actúa con base en un sistema de razonamiento.

La concepción de agente inteligente abre naturalmente la compuerta al estudio de los agentes en comunicación. Cuando existen varios agentes en un mismo entorno, es lógico 
pensar que ellos interactúen (Cohen \& Levesque 1995). Si se comunican, es posible que compartan metas o que estas entren en conflicto (Ekenberg, Roman y Danielson 1995). El conocimiento incorporado puede ser diferente o común y puede asumir diferentes formas de representación (Isasaki, 1995). La cooperación y la competencia comprometen las acciones de los agentes al igual que sucede en las sociedades naturales (Ito \& Yano 1995). Surge, entonces, la necesidad de estudiar la coordinación, como una forma de liderazgo entre los agentes artificiales autónomos (Osawa 1995). Dadas estas condiciones, la IA evoluciona en la perspectiva de la cognición social.

Si bien es cierto que el foco de la IA se centra en el agente inteligente, el estudio de los entornos tiene también su lugar y es especialmente interesante en una perspectiva de aprendizaje y de aplicaciones a la educación. Cuando se habla del mundo de los agentes artificiales, se está denotando un mundo restringido, un micromundo. Como tal es una entidad diseñada y de carácter formal. Es un sistema compuesto de objetos, relaciones entre objetos, operadores y operaciones que transforman los objetos (Thompson 1987). La formalización de la estructura del micromundo -parte declarativa- determina la existencia de un conjunto de leyes. las cuales están constituidas por el cuerpo de inferencias -teoremas- demostrables a partir de esta estructura. De manera similar al agente natural, el agente artificial debe actuar en coherencia con las leyes del micromundo para poder controlarlo. Parte de este proceso es el descubrimiento mismo de las leyes -aprendizaje-. El lenguaje Logo de Papert (1980) provee a la tortuga -aunque ésta no es un agente con capacidad perceptiva- de un entorno bidimensional en el cual se puede avanzar, retroceder o girar, dispone de un lápiz con el cual puede hacer trazos, allí, el circulo y las demás figuras geométricas bidimensionales obedecen a relaciones regulares que corresponden a una forma de geometríaa. En un entorno tridimensional podemos pensar en una cabina de avión en la cual un robot coloca cajas de diferentes tamaños y formas obedeciendo a relaciones de ubicación y orientación. Ambientes como éstos representan relaciones del mundo real con base en las cuales las personas razonan.

\section{Aproximaciones metodológicas de la Inteligencia Artificial}

Franklin (1995) hace algunas anotaciones que nos parece interesante resaltar en aras de ubicar los estudios de IA dentro de las dimensiones de lo posible, antes de caracterizar el cómo de los estudios que caben dentro dé los difusos límites de este campo. Estas son:

La inteligencia es un continuo que se muestra en los seres en grados, contrario a la forma del todo o nada.

- La inteligencia de un agente depende de la integración de componentes y se realiza, en la práctica, de manera parcial.

- La activación de la inteligencia se lleva a cabo por conjuntos de mecanismos que se ubican en un entorno.

- El fluido continuo de la acción absorbe totalmente la inteligencia: el sistema inteligente está permanentemente determinando qué hacer en seguida.

- La inteligencia opera sobre las sensaciones para generar información para su propio uso. 
- La inteligencia usa información almacenada en memoria para generar acciones mediante un proceso reconstructivo, más que por simple recuerdo.

- La inteligencia se puede representar de manera limitada e incremental: las máquinas del futuro serán más inteligentes que las actuales.

Si se sigue el enfoque clásico del test de Turing (1950), el desarrollo de sistemas inteligentes requiere como tarea fundamental identificar, adquirir y representar las manifestaciones de la inteligencia natural. Según Alíen Newell, uno de los fundadores del movimiento de Inteligencia Artificial, el principal problema que trata de resolver esta disciplina es el de la representación del conocimiento. En el denominado tercer debate (Franklin 1995) aparece una segunda posición derivada de los desarrollos de las tendencias conexionistas (Brooks 1991) que sostiene que los agentes pueden actuar sin representaciones. Los puntos críticos que no se resuelven en esta posición son: ¿necesita el programador representar estructuras para obtener inteligencia?, ¿cuándo un agente percibe forma representaciones? Las respuestas históricamente han sido positivas. De todas maneras es un debate que requiere de mayor evolución para llegar a un desenlace

La primera etapa de la construcción de agentes inteligentes tiene que ver con la adquisición y representación de las dimensiones de la inteligencia natural y la segunda con la creación de nuevas estructuras razonando a partir de mecanismos creados para imaginar dimensiones nuevas, pensamiento subjuntivo, en expresión de Hofstadter (1979). Dicho de otra manera. la creación de inteligencia artificial requiere una dinámica de modelamiento y otra de predicción a partir del modelo creado. Siguiendo el pensamiento de Hoftstadter, es posible crear un modelo de juego de un equipo de fútbol a partir de la observación sistemática de un conjunto suficiente de partidos jugados por el equipo de interés. Una vez creado, se puede entrar a analizar las posibilidades del modelo mismo, desprendiéndose de las particularidades del sistema que sirvió de base para el modelamiento. El resultado de este análisis muestra los alcances propios del modelo artificial

Gran parte de los estudios de IA se centra en procesos de solución de problemas como condición en la cual el conocimiento muestra todo su potencial. En el desarrollo de sistemas expertos, el ingeniero debe identificar el conocimiento utilizado y las estrategias que se siguen en la solución de problemas que caen dentro de la órbita de su especialización. Este proceso de adquisición requiere de métodos apropiados.

Una metodología caracterizada en el campo de la IA en funciones de adquisición de conocimiento es el análisis de protocolos de reportes verbales (Erickson y Simon 1993). Surge del trabajo de observación sistemática de sujetos jugando ajedrez o solucionando problemas de aritmética críptica (Newell y Simon 1972). Se orienta a identificar las secuencias de estados internos de la mente en el proceso cognitivo. Le da pie para afirmar que el lenguaje refleja de manera válida el pensamiento. La observación es intensiva sobre los procesos de cada sujeto y cifra su valor en la consistencia teórica del modelo de procesamiento de información que le sirve de base para la predicción como mecanismo fundamental de validación.

El modelo de procesamiento considera un ambiente o entorno de la tarea que entra en contacto con el sujeto a través de censores, un procesador, una memoria y mecanismos efectores. Los objetos del procesamiento de información son símbolos y con ellos se forman estructuras. La solución a un problema es una sucesión de transiciones de estado que, en su conjunto, determinan una transformación con un estado solución como fin. Una 
transición resulta de aplicar un operador a un operando. Un método es una lista de transiciones posibles. La percepción que el sujeto tiene de los estados posibles para la solución del problema constituye el espacio del problema y evoluciona en la medida que el sujeto intenta resolver el problema. Finalmente, la representación de este proceso simbólico constituye un programa para el procesador. Esta relación entre pensamiento y lenguaje simbólico es el vínculo directo entre los procesos de pensamiento natural y la programación artificial (Maldonado, 1992).

Un ejemplo ilustrativo de la actividad de adquisición y representación en IA y que utiliza el método de análisis de protocolos es el traído por Glancey (1987) y que se expresa en los siguientes pasos:

A. Discusión informal de un caso previamente diagnosticado por Mycin.

B. El experto resuelve un caso mientras el experimentador pregunta sobre el razonamiento que sigue.

C. Se le pide al experto que describa un caso típico para cada una de los diagnósticos principales.

D. Se le pide al experto que presente un caso al experimentador Inverso de B.

E. Los mismos casos discutidos en B y D se presentan a diferentes expertos.

F. El modelo de estrategia desarrollado se presenta al primer experto para su evaluación.

G. Los mismos casos son presentados a los mejores estudiantes del experto.

H. Se discute cada regla con el experto y se agrupan de acuerdo con las hipótesis que sustentan.

En la aproximación metodológica de la IA algunos conceptos son fundamentales en el proceso de adquisición de conocimiento (Maldonado, 1994): el ambiente de la tarea, el espacio del problema, los métodos de solución, la memoria de trabajo y la memoria de largo plazo. Estos conceptos denotan fenómenos que interactúan de manera dinámica en el proceso de razonamiento.

El entorno presenta al sujeto las variables del problema a través de los mecanismos aferentes y se denomina ambiente de la tarea. La solución de un problema se lleva a cabo por una sucesión de estados. Esta sucesión de estados se denomina espacio del problema y asume dos acepciones: el espacio lógico del problema, y el espacio psicológico. El primero esta conformado por los posibles estados que pueden entrar en el proceso de solución. El segundo es el conjunto de estados en el campo perceptivo del sujeto en cada momento de la solución y que evoluciona a medida que se desarrolla el proceso. El paso de un estado a otro se hace mediante la aplicación de operadores a objetos. Un estado se transforma en un sucesor; esta sucesión de operaciones constituye la estrategia o método de solución del problema. Las estrategias usadas dependen de información procedimental almacenada en memoria de largo plazo, la cual se enriquece con el feedback del proceso. En cada momento de éste, la información tanto de los objetos, como de los operadores y procedimientos están presentes al solucionador en lo que se denomina memoria de corto plazo o memoria de trabajo. 
La información recogida a través del proceso de adquisición de conocimiento tiene su equivalente en el proceso de simulación, razón por la cual se han desarrollado técnicas paralelas a nivel de programación que se preocupan por los temas del entorno, (ej. los micromundos), aprendizaje y percepción (ej. redes neuronales, reconocimiento de patrones, procesamiento de lenguaje natural, interfaz hombre-máquina) memoria (estructuras de datos, bases de conocimiento, redes semánticas, grafos conceptuales), y la representación del espacio y el concepto de espacio del problema se convierte en pieza fundamental del proceso de representación (al decir de Winston, 1992) la representación adecuada del espacio del problema es la parte fundamental en su solución y sobre éste o se diseñan los procesos de búsqueda).

\section{Inteligencia Artificial y Educación}

El hecho de que la IA tenga como objeto primordial de estudio el conocimiento y su forma de representación hace que desde sus inicios surjan las preguntas relacionadas con el aprendizaje y la educación. Newell y Simón (1972) analizan en profundidad las características de los expertos solucionando problemas frente a los aprendices. Pero, la proyección más destacada de la Inteligencia Artificial a la educación se da con los Tutores Inteligentes (Barr y Feigenbaum, 1981). Esta tendencia de pensamiento establece una dinámica de generación de modelos cognitivos con validación a través de mecanismos computacionales (Maldonado, 1992).

Una revisión de la amplia gama de tópicos que han estado dentro de la mira de los investigadores sobre tutores inteligentes es presentada por Wenger (1987). En los tutores inteligentes hay temas medulares: el dominio de conocimiento, la pericia pedagógica, el modelamiento del estudiante y su proceso de aprendizaje, y la dinámica comunicacional.

Los tutores inteligentes (TI) se muestran como productos tecnológicos, pero, su valor no está tanto en la comercialización de los programas que ha sido muy reducida, sino en los aportes a la metodología de estudio de los procesos de aprendizaje y a la formación de un enfoque cognitivo y computacional de la pedagogía. Estas investigaciones, que no han sido independientes de otras áreas de aplicación de la IA, han contribuido al desarrollo de software más amigable en la industria misma.

La temática de los TI ha motivado estudios más independientes de temas específicos a través de procedimientos de modelamiento. Tal es el caso de la metacognición (Nelson T. O., \& Narens, L. 1990)., el error de los estudiantes en el proceso de aprendizaje (Wenger 1987), procesos de feedback y sus implicaciones en el diseño de materiales educativos (Fisher y Handl 1988), el uso de la analogía y la metáfora en el aprendizaje (Streitz, 1988), los procesos de evaluación y tutoría (Johnson and Soloway, 1987), las creencias y prejuicios y su influencia en la toma de decisiones de los estudiantes (Sleeman, 1987).

En un contexto más reciente, es importante destacar las repercusiones de trabajos más amplios dentro de la ciencia cognitiva. Los trabajos de Howard Gardner, al tiempo que caracterizan toda una corriente de pensamiento, muestran la perspectiva de las múltiples dimensiones de la inteligencia humana. Recientemente se inicia el estudio de diferentes formas de razonamiento como es el caso de la imaginería mental y la imaginería computacional, el razonamiento analógico, la simulación de paradigmas científicos y su aplicación al aprendizaje de las ciencias, el razonamiento espacial cualitativo y cuantitativo y el razonamiento temporal (Glasgow et al. 1996). Finalmente cabe destacar la relación entre estructuras de software y la aplicación de técnicas de representación de 
conocimiento, tomando como punto de referencia el sistema sensorial del usuario y SU forma ce procesar información. Tal es el caso de los hipertextos, los hipermedia, la realidad virtual y la telemática. Sobre el tema se vienen publicando muchos trabajos, como quiera que se relaciona con avances tecnológicos muy recientes. La asociación entre modelos conexionistas de representación y los hipertextos e hipermedia se ha destacado en estudios como los de Jonassen, al tiempo que se abre una relación con el constructivismo en educación. En Maldonado (1996) se utilizan los mapas conceptuales como mecanismos de evaluación de relaciones conceptuales. El impacto sobre el sistema perceptivo y consecuentemente sobre el conjunto del proceso de información -memoria, razonamiento, feedback y formación conceptual- de la hipermedialidad es un tema de muy reciente investigación. Landow (1992) presenta una perspectiva epistemológica de las transformaciones predecibles a partir de la hipertextualidad. La relación en inteligencia artificial con los trabajos de Imaginería mental e imaginería computacional establecen una relación muy estrecha entre lo hipermedial, lo virtual y los procesos de percepción, representación y razonamiento.

La telemática, por otra parte, se proyecta con potencialidades educativas relacionadas con el ideal de convertir la sociedad en su totalidad en una aldea del aprendizaje. Por su reciente incursión en la sociedad sus perspectivas son más predichas que evaluadas (Steinberg, 1992), sin embargo, algunos problemas son de consideración de la IA. La riqueza de información sobrepasa la capacidad de procesamiento del usuario de manera abrumadora, lo cual hace que se presenten problemas relacionados con el acceso, jerarquización, actualización y valoración. La superación de la limitante de la distancia, a lo cual responde la telemática, abre temas de investigación como el trabajo colaborativo y los procesos de diálogo en la educación a distancia (Verdejo, 1994)

Finalmente, vale destacar, por las proyecciones que se tienen en la línea de investigación objeto de este trabajo, la relación entre Inteligencia Artificial y lúdica (Maldonado, 1 996a). Los trabajos clásicos iniciales de la IA utilizaron juegos como el ajedrez y las damas como condiciones propicias para el estudio de solución de problemas. En razonamiento espacial juegos como el Pentominó y el Tetris (Kirsh, \& Maglio, 1994) constituyen condiciones de valor especial para entender la dinámica del razonamiento cualitativo.

\section{Importancia de la inteligencia artificial en la enseñanza de las ciencias}

Las ciencias constituyen una fuente inagotable de problemas de investigación para la mente humana. La solución de estos problemas requiere formas diversas y elaboradas de razonamiento que constituyen un reto para quien se dedica a estudiar su aprendizaje. Los métodos de trabajo de la Inteligencia Artificial pueden dar aportes valiosos tanto en la formalización de modelos pedagógicos que permitan comprender los procesos de solución de problemas, como en la generación de ambientes computacionales que faciliten el aprendizaje. Como ejemplos ilustrativos de esta aproximación se puede considerar la simulación de formas de razonamiento tales como espacial, temporal, analógico y basado en contextos, el aprendizaje a partir de casos, el diseño de sistemas basados en metáforas, el modelaje de estudiantes y el desarrollo de tutores inteligentes, de ambientes virtuales de investigación y de micromundos de aprendizaje, entre otros 


\section{Antecedentes de la línea de doctorado}

La línea de investigación que se propone se ubica en el contexto de los estudios de Inteligencia Artificial orientados a la representación de diferentes formas de conocimiento y a la comprensión de los procesos de aprendizaje y formación de la persona. En las universidades participantes se vienen adelantando trabajos que comparten enfoques básicos fundamentales que dan base para hablar de la línea de Inteligencia Artificial.

A continuación se caracterizan los trabajos desarrollados en la Universidad Industrial de Santander y en la Universidad proponente de la línea.

\section{La investigación desarrollada por el grupo de la Universidad Industrial de Santander}

Se ha centrado en la definición de las características educativas y tecnológicas que deben reunir los materiales educativos informatizados (MEI) para que promuevan y acompañen procesos de aprendizaje. Se ha reflexionado sobre modelos pedagógicos cuyos lineamientos puedan proyectarse a través de MEI de tipo inteligente. En esta lógica se estudia el proceso de representación de conocimiento y lineamientos provenientes de teorías del aprendizaje. Se han hecho desarrollos en las áreas de lenguaje, matemáticas y sociales para el Sistema Formal de la Enseñanza Básica y en las áreas de estadística y física en educación superior. Este trabajo está siendo enriquecido con los aportes de los docentes de la Especialización en Pedagogía Informática, la cual se orienta al uso óptimo de la informática para promover y acompañar el aprendizaje.

La base cognitiva de estos trabajos la constituyen estudios sobre representación de conocimiento, solución de problemas y construcción de conceptos en las áreas en las cuales se trabaja.

Los desarrollos en programación declarativa, programación orientada a objetos, multimedia y técnicas de representación del conocimiento constituyen su base tecnológica.

La Universidad Industrial de Santander lideró la constitución de la Red de Modelamiento Sistémico que constituye uno de los apoyos potenciales del doctorado, por el nivel académico de los miembros de esta red.

\section{EI Proyecto TECNICE de la Universidad Pedagógica Nacional}

El antecedente inmediato de la Línea de Inteligencia Artificial y Comprensión de Procesos de Razonamiento en Ciencias es el proyecto TECNICE -Tecnologías de la Información y la Comunicación en Educación- (Maldonado, 1991a). Este proyecto, a su vez, surgió como resultante de un conjunto de investigaciones y programas académicos desarrollados durante las décadas de los setenta y ochenta y que confluyen en una tendencia de pensamiento que define la Pedagogía como ingeniería social con tres grandes tipos de actividades: caracterización, proyección e intervención del aprendizaje y la cultura (Maldonado 1986: Maldonado, 1996). 


\section{Caracterización del aprendizaje y la cultura}

La sicología en su evolución ha contribuido substancialmente a la comprensión del proceso de aprendizaje como dimensión eminentemente individual. La antropología y la etnoinvestigación, por otra parte, dilucidan la cultura como una formación social, donde el aprendizaje es característica compartida por miembros de las comunidades y que se manifiesta en hábitos, formas de interacción social, actitudes y paradigmas cognitivos condicionantes de las diferentes formas de juicio y decisión

El interés investigativo se orientó hacia la dinámica de interacción, los entornos y las características culturales de los actores del proceso educativo. En Betancourt y otros (1974), con base en la metodología de historias de vida, se estudian dimensiones culturales de maestros y estudiantes colombianos y se elabora el concepto de cultura del salón de clase' como una formación institucional cerrada a la información y, por tanto, con puntos de equilibrio muy estables. Estos trabajos están en relación con el movimiento latinoamericano que más explícitamente resalta la dimensión cultural de la educación, y que, a nuestra manera de entender, está representada por el pensamiento de Paulo Freire (1970). Esta tendencia de corte etnográfico se proyecta luego a la caracterización de los procesos de interacción en ambientes enriquecidos con tecnologías de la información (Maldonado, 1991 b), siguiendo una corriente de pensamiento representada por los trabajos de Sherry Turkle (1984)

La caracterización cultural se desarrolla desde la investigación cuantitativa con métodos estadísticos aplicados a la evaluación de actitudes de los actores del escenario educativo. Los valores son parámetros condicionantes del procesamiento de información y del aprendizaje, en especial, en la medida en que son compartidos por educandos y educadores. En la perspectiva de kerlinger (1975), quien estudia las actitudes de los educadores frente al cambio, se aplicó la metodología $Q$ con el objeto de analizar jerarquías de valores de estudiantes y profesores (Gómez et al., 1985), encontrando tendencias contrarias en los dos grupos de sujetos.

\section{Las estrategias de intervención}

Los programas de Magíster en educación de la Universidad Pedagógica Nacional ofrecieron condiciones propicias para el desarrollo de trabajos en la perspectiva de evaluar los procesos de aprendizaje en función de los entornos y sus interacciones mediadas por materiales educativos como mecanismos de transmisión de información. En Maldonado y Sequeda (1974) se analiza cómo la estructura de un ambiente de aprendizaje incide en el desarrollo de hábitos, en términos tanto de la duración como en la eficiencia de las sesiones de estudio libre, de niños de cuarto grado de primaria. Luego, la comparación de métodos de enseñanza con filosofía de dominio mostró que el uso de materiales textuales genera diferencias significativas en el aprendizaje de la psicología en los primeros cursos de licenciatura y cómo los procesos de evaluación son factores críticos en el desarrollo de la capacidad de aprender (Maldonado et al. 1977). Estas investigaciones generaron interés por el aprendizaje dirigido por el mismo estudiante, primero en la perspectiva del autocontrol (Kanfer y Philips 1970) y luego en el marco de la metacognición (Derry y Murphy, 1986).

El estudio de la incidencia de ordenadores de navegación, como son los objetivos, los índices y los mapas instruccionales, sobre la toma de decisiones en la navegación a través de un hipertexto, y la autoevaluación del proceso de aprendizaje (Maldonado, 1986) sirve de base para la introducción del computador como ambiente de 
experimentación y de técnicas de inteligencia artificial para la representación de conocimiento. Este proyecto abrió el camino al estudio del proceso de autoevaluación en la solución de problemas y en la comprensión mensajes hipertextuales usando como dominio de aprendizaje el diseño industrial (Maldonado y Andrade, 1996) y, al análisis de la autoevaluación como activador de estrategias de solución de problemas y el uso de simuladores como dispositivos para el entrenamiento de profesores que desarrollen habilidades para enseñar por procesos.

Los trabajos de los profesores tuvieron eco en el desarrollo de investigaciones para llenar el requisito de tesis de Magíster. En ellas se muestran tendencias de pensamiento que han perdurado en el proyecto TECNICE. Se estudió el proceso de solución de problemas en niños preescolares (Monroy y Terrero), iniciando un interés por el estudio del pensamiento dentro del marco de la teoría del procesamiento de la información. Un conjunto de trabajos se orientó a la evaluación del valor de los materiales educativos en términos de ganancia de información (Larrota y Morales (1984), Vega (1984)), apoyándose en la teoría matemática de la información (Ashby 1963). Se evaluó el nivel de comprensibilidad de textos escolares (Mogollón, Muñoz y Oviedo (1984)) y metodologías para mejorar la comprensión de mensajes textuales (Hernández, 1984). Se desarrollaron estrategias para identificar la efectividad de sistemas de información institucional (Villela, 1984) y se estudió los procesos de toma de decisiones en la elección de profesión (Velazco, 1984).

\section{La proyección}

TECNICE concreta un ideal de proyección cultural y el inicio de una serie de actividades de prospectiva pedagógica. Con la concepción de que a través de los proyectos se pueden crear realidades futuras como resultado de cadenas de transformaciones de la realidad presente, se diseñó el proyecto $y$, luego, se pasó a su puesta en operación y gestión. En el marco de TECNICE, los trabajos de investigación deben confluir en proyectos tecnológicos como solución a problemas identificados en alguna realidad concreta.

Una primera meta fue la de construir un entorno propicio a la investigación y al desarrollo de proyectos en el campo de las tecnologías de la información y la comunicación para el sector educativo, conscientes del papel determinante de los ambientes en el desarrollo del aprendizaje y de la actividad productiva de los grupos. En la estrategia para conformar un grupo de investigación se ideó un esquema de juego para los jugadores reales, no para los ideales. La preocupación central estuvo en la formulación de unas metas y problemas que crearan tensión teleológica y en la creación de un escenario tecnológico donde investigadores y académicos pudieran desarrollar juegos de imaginación para responder a esta dinámica. En principio, cualquier idea, por especulativa que aparezca, es bienvenida, con tal de que dé origen a una representación en un programa de computador, en un objeto visible o en procedimientos que generen procesos reales.

Las líneas de trabajo de TECNICE han sido: investigación y desarrollo de software, formación, capacitación, telemática, multimedia y gestión.

En investigación y desarrollo de software se han adelantado proyectos sobre metacognición, razonamiento espacial y enseñanza de las ciencias sociales. Maldonado y Andrade (1996) analizan el impacto de ambientes computacionales que propician juicios acerca de la facilidad, la adquisición y la sensación de aprendizaje - metamemoria - sobre 
el aprendizaje del razonamiento espacial; por otra parte, estudia la relación entre la activación de estrategias previamente a la solución de problemas presentados por el computador y el desarrollo de habilidades de solución de problemas; y se interesa por ver el efecto del entrenamiento de profesores basado en el estudio de simuladores de estrategias de solución de problemas sobre la orientación de su actividad docente.

Vargas et al. (1996) desarrollan un trabajo sistemático de diseño, producción y validación de hipertextos en ciencias sociales con temas como los derechos humanos, estado y sociedad civil $\mathrm{y}$, pensamiento político democrático. El interés por la representación hipermedial del dominio del conocimiento se combina con el interés por el trabajo interdisciplinario y la educación de líderes comunitarios.

En los programas de formación se priorizan dos tendencias; la proyectual, vinculada al diseño y producción de software para educación, a través del programa de especialización y la investigativa, apoyada en el desarrollo de estudios orientados a vincular las tecnologías de la información a la comprensión de procesos culturales y de aprendizaje, a través del programa de Magíster. En esta dinámica se han producido paquetes de software para las diferentes áreas de la enseñanza en educación básica y vocacional y se han desarrollado trabajos de investigación en temas como la dinámica de sistemas y la enseñanza, el modelamiento y el razonamiento espacial, razonamiento sobre mecanismos, generadores de problemas y desarrollo del pensamiento divergente, lectura y escritura en los hipermedia, metacognición y educación especial, procesos cognitivos en la navegación hipertextual, hipersonido y razonamiento espacial en personas con deficiencia visual, razonamiento analógico y aprendizaje de la matemática, razonamiento no monótono en toma de decisiones de orientación profesional, impacto cognitivo de metodologías de formación a medida en temas de informática, impacto cognitivo de la construcción de mapas conceptuales apoyados por computador.

La experiencia vivida durante esta década en TECNICE da pie para sustentar la hipótesis de que los ambientes de aprendizaje en si mismos operan como transformadores del razonamiento lógico en discernimiento moral, de la especulación en objetivación y de la objetivación en imaginación.

En el seno de TECNICE se ha desarrollado un diálogo efectivo entre tendencias conceptuales y teóricas y entre metodologías de investigación. Se ha buscado intencionalmente la complementaridad antes que la oposición, dando como resultado riqueza de visiones y trabajos interdisciplinarios que convergen en objetivos y productos. Las aproximaciones más favorecidas han sido: la etnometodología la metodología experimental, análisis de protocolos de informes verbales y el modelamiento sistémico

El interés del grupo TECNICE ha sido el de modelar el mundo de la educación mediante un juego dinámico entre los enfoques cualitativos y cuantitativos. El estudio de lo metacognitivo realza la autonomía como dimensión básica del trabajo creativo. En la práctica se integran intereses lógicos, estéticos, éticos y lúdicos en la generación de soluciones informáticas para la educación.

\section{La línea dentro del área de enseñanza de las ciencias}

La línea es parte integral del Área del Programa de Doctorado interinstitucional en Educación denominada de Enseñanza de las Ciencias, la cual temáticamente gira en torno al desarrollo cognitivo manifestado en la formación de conceptos y preconceptos y en los procesos de razonamiento complejo y solución de problemas. El diálogo 
permanente entre las líneas que componen el área potencia un desarrollo científico que se vislumbra promisorio. Las relaciones de complementaridad se perciben, ubicados en la Línea de Inteligencia Artificial y Comprensión de Procesos de Razonamiento, desde las perspectivas: temática, paradigmática, metodológica y técnica. Desde la perspectiva temática, la línea 3 de Solución de Problemas, dirigida por la Doctora Margie Jessup, y la línea 5 de Evaluación de los Procesos de Razonamiento Complejo, dirigida por el Dr. Fidel Cárdenas, ambas enfocadas en una perspectiva cognitiva, contribuyen substancialmente a delimitar el objeto de investigación.

Desde la perspectiva paradigmática la Línea 2 de Elaboración de Conceptos científicos dirigida por el Dr. Fabio Vélez permite ver las formas de razonamiento como procesos que adquieren características resultantes de la evolución de las ciencias. El concepto de paradigma, como parámetro básico de la percepción y el razonamiento, se estudia en su dimensión epistemológica e histórica.

El aprendizaje de las ciencias se ve desde la dinámica de las relaciones del enseñante y el aprendiz en una perspectiva cultural en la cual los preconceptos, los conceptos, el sentido común y las expresiones en formato de las ciencias entran en interacción y condicionan la forma de razonamiento. Múltiples agentes inteligentes entran en interacción en la dinámica de la cultura. Este tipo de relaciones es aporte muy relevante de la línea 1 dirigida por los Drs. Lilia Reyes y Luis Enrique Salcedo y la línea 6 dirigida por el Dr. Alfonso Claret.

Desde la perspectiva metodológica, la línea 1 profundiza en enfoque etnográfico, base de la comprensión de la dinámica cultural, la línea 2 en el análisis histórico y epistemológico, la línea 5 sobre diseños experimentales estadísticos.

La línea de inteligencia artificial se en-foca temáticamente sobre procesos de razonamiento de orden cualitativo, se complementa conceptual y metodológicamente de las aproximaciones de las otras líneas y da aportes sobre métodos de adquisición de conocimientos, como es el caso de la metodología del análisis de protocolos de informes verbales y métodos de representación de conocimiento y simulación. En una perspectiva epistemológica presenta aportes desde la teoría de sistemas y desde las formas de representación del conocimiento. Sus aportes técnicos contribuyen tanto a la sistematización de los ambientes experimentales como al procesamiento de información de las investigaciones.

\section{Estructura de la línea de investigación}

Se concibe el doctorado como un nivel de formación altamente especializado, muy centrado en la investigación personal, curricularmente flexible y con fuertes exigencias en cuanta consistencia conceptual y metodológica. El proceso se nutre de un diálogo intensivo entre pares y dentro de una comunidad científica. Por esta razón, se preven procesos de concertación de temáticas y experiencias curriculares.

La dimensión propositiva inicial de esta línea contemplo, en razón de los antecedentes que la motivan, el estudio del aprendizaje autodirigido y los procesos metacognitivos, el razonamiento cualitativo con manifestaciones en e> razonamiento espacial, la imaginería mental, el uso de analogías en el aprendizaje a partir de casos, la metáfora como vinculo entre experiencia previa y el aprendizaje y la toma de decisiones con información incompleta (razonamiento no-monótono). 
Su desarrollo considera tres componentes básicos: la representación del conocimiento mediante modelos, el uso de metodologías consistentes de validación de la teoría y el desarrollo de dispositivos computacionales como mecanismo de simulación.

A nivel teórico interesa el estudio, simulación y comprensión de procesos de razonamiento cualitativo.

A nivel metodológico se basa en enfoques que permiten el estudio de procesos cognitivos y el diseño y desarrollo de sistemas basados en conocimiento con proyecciones al campo de la educación.

A nivel técnico los acercamientos se basan en diferentes formas de lógica y razonamiento cualitativo y cuantitativo y paradigmas de programación, como es el caso de la programación declarativa y orientada a objetos.

La línea comparte la estructura general del área en la cual se consideran tres dimensiones generales: humanística, metodológica y pedagógica

En la dimensión humanística, la línea recibe aportes de las otras líneas y, a su vez, aporta una reflexión epistemológica y educativa sobre los siguientes aspectos:

- Ciencia cognitiva e inteligencia artificial: Son muchos los trabajos que se pueden citar a este respecto. Las obras de Franklin (1995) y Hostadter (1979) presentan un panorama global y dinámico de los desarrollos de la inteligencia con proyecciones a lo epistemológico e interdisciplinar.

- Teoría general de sistemas, cibernética y sociedad: Trabajos de Von Bertalanfy y Luhman pueden ser base para organizar seminarios y ensayos.

En la dimensión estratégico-metodológica de la investigación comparte actividades con las otras líneas del área y enfatiza en el estudio de Metodologías de investigación en Inteligencia Artificial y Educación tales como:

- Análisis de protocolos: El trabajo más organizado y que al mismo tiempo ayuda a profundizar sobre el paradigma del procesamiento de información es Erikson, K. A. and Simon, H. A. (1993). A partir de este trabajo se puede analizar muchas investigaciones recientes. La revista Cognitive Science y Cognitive Instruction provee trabajos de excelente calidad en esta dirección.

- Tendencias etnográficas: se partirá de las orientaciones de las líneas de Reyes y Salcedo.

- Tendencias estadísticas: Cohen (1997) presenta una visión actualizada de los problemas metodológicos propios de la Inteligencia Artificial. A partir de esta obra se pueden desarrollar ampliaciones, según las exigencias de los proyectos de investigación.

En la dimensión pedagógica, a su vez, comparte con las otras líneas y aporta un estudio especifico sobre temas como los siguientes:

- Ciencia cognitiva y pedagogía: Gardner (1993) se orienta a dimensiones específicas de la inteligencia que tocan con los espacial y lo cualitativo. 
- Inteligencia artificial en educación: Wenger (1987) es una revisión con una estructura que facilita la incorporación de investigaciones más recientes.

- Hipertexto, hipermedia, Realidad virtual y aprendizaje: Landow (1992) presenta un esquema de análisis con capacidad de incorporar nuevos desarrollos y perspectivas.

- Telemática y aprendizaje colaborativo. Proceedings of the First International Conference on Multiagent Systems ICMAS-95 y Trabajos de Verdejo pueden servir de base para la discusión de un tema que está en proceso de sistematización.

\section{Dimensiones específicas de la línea}

Las investigaciones de los doctorandos se fundamentan en campos especializados con proyecciones teóricas y metodológicas. A continuación se presentan temas centrales de estudio y desarrollo acompañados de una referencia bibliográfica como base para la orientación temática y la definición de las actividades curriculares que se llevaran a cabo.

\section{a. Lógica y representación del conocimiento}

Teoría de los agentes inteligentes. La obra de Russell, S. y Norvig, P. (1996) presenta una panorámica bastante completo de la IA en la perspectiva de los agentes inteligentes, al tiempo conceptual y metodológica en cuanto a representación del conocimiento. A partir de sus planteamientos se pueden organizar actividades curriculares pertinentes.

- Programación lógica. La obra Sterling y Shapiro (1986) presenta una panorámica rigurosa y amplia que contribuye a la formación avanzada del programador.

- Modelos conexionistas de representación. La obra general de Rich, E. y Knight, K. (1996) y el trabajo más especializado de Sowa, J. (1984) pueden tomarse como punto de partida.

- Razonamiento espacial. El trabajo de Hernández (1994) complementado con Glasgow, Janice, Narayanan, N, H. and Chandraskaran, B. (1996) permiten una visión del razonamiento espacial vinculado al cuerpo de investigación sobre imaginería mental.

- Razonamiento basado en casos: Leake. David B. (1996) presenta una excelente compilación de trabajos que pueden fundamentar los inicios de seminarios y el desarrollo de investigaciones en esta dirección

- Razonamiento temporal: Bolc, Leonard, Szalas. Andrzej (1995) presentan una compilación de modelos formalizados y de aproximaciones teóricas con una amplia panorámica sobre el tema.

- Algoritmos genéticos: Mitchel, Melanie (1996) constituye una buena sistematización de esta corriente específica de pensamiento

\section{b. Paradigmas de programación}

Cada uno de los tópicos considerados como base tienen una plataforma de programación como representativa de la tendencia. El doctorando debe demostrar 
capacidad de desarrollo eficiente de sistemas basados en al menos una de estas plataformas.

- Programación orientada a objetos: Java o Toolbook.

- Programación visual: Acroscience.

- Programación declarativa: Prolog.

- Tecnología multimedial y realidad virtual: MicroMind Director.

- Tendencias en desarrollo de software en educación.

\section{c. Dominio de Conocimiento}

El estudio de una disciplina científica y. dentro de ella, de un conjunto específico de problemas, da origen al objeto de conocimiento al cual se aplica los procedimientos y técnicas de la inteligencia artificial. En el enfoque de doctorado que se presenta inicialmente interesan dominios de la biología, la física y la química, y el diseño, por la existencia de doctores en estas áreas participando en el área de doctorado. Sin embargo, esto no significa cierre a dominios del saber en los cuales se pueda contar con expertos y propuestas de investigación bien definidas

\section{REFERENCIAS BIBLIOGRÁFICAS}

Antonieu, G (1997). Non monotonic reasoning. Cambrige: AAAI Press/ The MIT Press, 6572.

Ashby, R. (1963). Introducción a la Cibernética. Editorial Nueva Visión.

Barr A. \& Feigenbaum, E. A (1981). The handbook of Artificial Intelligence. Los Altos CA: William Kaufmann, Inc.

Bolc, Leonard, Szalas, Andrzej (1995). Time and Logic: a computational approach. London : UCL Press Limited.

Boole, G. (1847). The mathematical Analysis of Logic. Cambridge: MacMillan, Barclay \& MacMillan.

Boole, G. (1954). An investigation of the laws of thought. London: Walton \& Maberly.

Brooks, R. (1991). Intelligence without representation. Artificial Intelligence, 47: 139-159.

Cohen, P. and Levesque H. (1995). Communicative actions for artificial agents. Proceedings of the First International Conference on Multiagent Systems ICMAS-95. Camnrige: AAAI Press/ The MIT Press, 65-72

Derry, S.J. and Murphy, D. A (1986). Designing systems that train learning ability: from theory topractice. Review of Educational Research. 56(1), 1-39. 
Ekenber, L , Roman M. and Danielson. M. (1995). A tool for coordinating autonomous agents with con flicting goals. Proceedings of the First International Conference on Multiagent Systems ICMAS-95. Cambrige AAAI Press/ The MIT Press, 65-72.

Erikson, K. A. and Simon, H. A. (1993). Protocol Analysis: verbal reports as data. Cambridge. The MIT Press. Second Edition.

Euler. L. (1735). The seven bridges of Konigsberg. In Newman, J.R. (1956). The world of mathematics. New York: Simon and Shuster.

Feigenbaum, Edward A. \& Feldman, Julian (1995) Editors. Computers \& Thought. Cambridge, Massachusetts. AAAI Press/ The MIT Press.

Fisher, P.M. and Handle, H. (1988). Improving of the a cquisition of knowledge by iriforming feedback. En Handl, $\mathrm{H}$. and Lesgold, A. Learning issues for intelligent tutoring systems. N.Y. Springer-Verlag.

Fortier, Paul J. (1986). Designing of distributed operating system: concepts and tehcnology. New York: McGraw Hill Book Company.

Franklin, Stan (1995). Artificial Minds. Cambridge, Massachusetts: The MIT Press.

Frege, G. (1879). Begriffsschnft. eme der arithm atisben nachgebildete Formelsprache des reinen Denkens. Halle: L. Niebert.

Fraga. G. (1884). Die Grundlagen der Arithmetic. Breslau: W. Koeber.

Freire, Paulo (1969). La educación como práctica de la libertad. Traducción del Portuguez. Educaçao como prática da libertade. Montevideo, Uruguay: Editorial Tierra Nueva.

Freire, Paulo (1970). La pedagogía del oprimido. Traducción del Portuguez. Montevideo, Unruguay: Editorial Tierra Nueva

Gardner, H. (1995). Estructura de la mente: La faena de las inteligencias múltiples. México: Fondo de Cultura Económica (segunda edición). Traducción del Inglés. Frames of Mmd: The theory of multiple intelligences. New York: Basic Books, 1983.

Gardner, Howard (1993. Arte. Mente y Cerebro. Buenos Aires: Editorial Paidós), reimpresión.

Glancey, W. J. (1987). Methodology for building an nelligent tutoring system. In Kearsley, G. P. Artificial intelligence \& instruction. Reading, Massachussetts: Addison-Wsley Publishing Company.

GLASGOW, J. \& PAPADIAS, Dimitri (1992). Computational Imagery. Cognitive Science, 16, 355-394.

Glasgow, Janice, Narayanan, N, H. and Chandraskaran, B. (1996) Editor. Diagramatic Reasoning: cognitive and computational perspectivas. Cambrídge, Massachusetts: AAAI Press/ The MIT Press. 
GOEL, V. \& PIROLLI, P.(1992). Structure of Design Problem Spaces. Cognitive Science. Vol 16, No 3, Jul.- Sep. pp. 395 - 429.

Gómez G., E., Maldonado G., L. F. y Vanegas, B. (1985). Autoridad, Libertad y Aprendizaje: Valores de docentes y discantes en la educación. Investigación Educativa, No 1.

Hernández, D. (1994). Qualitative representation of spatial knowledge. N.Y.: SpringerVerlag.

Hostadter, D. (1979). Gódel, Eschery Bach. New York: Basic Books.

Isozaki, H. (1995). Reasoning about belief basad en common knowledge of obsetvability of actions. Proceedings of the First International Conference on Multiagent Systems ICMAS-95. Cambrige: AAAI Press/ The MIT Press, 65-72.

Ito, A. and Yano, H. (1995). The emergence of cooperation in a society of autonomous agents - the prisoner's dilemma gama:Under the disclosure of contract histories. Proceedings of the First International Conference en Multiagent Systems ICMAS-95. Cambrige: AAAI Press/ The MIT Press, 65-72

Johnson, W.L. and Soloway, E. (1987). Proust: an automatic debugger for pascal programs. En Kerasley, G.: Artificial intelligence \& instruction: applications and methods. Reading, Massachussetts: Addison-Wesley Publishing Company.

Kanter, F. H. and Phillips, J. 8. (1970). Leaming foundations of be ha vior therapy. New York: John Wiley \& Sons, Inc.

Kerlinger, F. (1975). investigación del comportamiento. México: Editorial Interamericana.

Kirsh, David \& Maglio, Paul (1994). On distinguishing epistemic from Pragmatic Action. Cognitive Science_18(4), 513-594.

Kowalski, R. (1986). Lógica, programación e inteligencia artificial. Madrid: Editorial Díaz de Santos. Traducido del Inglés: Logic for problem solving.

Landow, G. (1992). Hipertexto: la convergencia de la teoría crítica contemporánea y la tecnología. Barcelona: ediciones Paidós.

Leake, David B. (1996) Editor. Case based reasoning : experiences, lessons \& futura directions. Cambridge, Massachusetts: AAAI Press/ The MIT Press.

Lugger, F.L. and Sutbblefield, W.A.(1993). Artificial Intelligence: .Structures and strategies for complex problem solving. Redwood City, CA: The Benjamin/ Cummings Publishing Company, Inc, Second Edition.

Maldonado G., L. F. (1986). La pedagogía y la función social del pedagogo. Revista Acción Educativa No 7.

Maldonado G., L. F. (1991b). Procesos de interacción en un aula computarizada. Informática Educativa, 4,1, 44-60. 
Maldonado G., L. F. (1992). Hipertexto: una proyección educativa basada en el ambiente HyperCard-HyperTalk-HyperTalk: Publicación Universidad Pedagógica Nacional.

Maldonado G., L. F. (1992). Modelos cognitivos e informática. Memorias del Congreso Nacional de Informática Educativa. Bogotá.

Maldonado G., L. F. (1992). Programación Lógica: Publicación Universidad Pedagógica Nacional.

Maldonado G., L. F. (1994). Análisis de Protocolos: una alternativa metodológica para el estudio de procesos cognitivos en personas con discapacidad. Publicación Universidad Pedagógica Nacional.

Maldonado G., L. F. (1994). Diseño, Administración y Desarrollo de Proyectos en Educación. Publicación Universidad Pedagógica Nacional.

Maldonado G., L. F. (1995). Creación de Hipertextos. Publicación ICFES y Universidad Javeriana.

Maldonado G., L. F. (1996a). Desarrollo Cognitivo, Informática, Lúdica y. ACUC: Noticias Informáticas, No 161, 39-41.

Maldonado G., L. F. (1996b). La pedagogía como Ingeniería social. Revista de Pedagogía, No 14-15 Facultad de Educación, Universidad de Antioquia.

Maldonado G., L. F. (1996c). Learner Controlled, Computar-Basad Environment Fon Developing Design Capabilities. Second Jerusalem International Science \& Technology Education. Conference JISTIC 96. Proceedings.

Maldonado G., L. E. y Sequeda, M. (1974). Duración y eficiencia de la conducta de estudio en el aprendizaje de matemáticas a nivel de cuarto grado de primaria. Tesis de grado Universidad Pedagógica Nacional.

Maldonado G., L. E. y Vargas O., G. (1994). Analogía, Metáfora y Análisis de Protocolos. En memorias de la Primera Conferencia Colombiana Sobre Modelamiento Sistémico. Universidad Industrial de Santander.

Maldonado G., L. F., Cuan A., L. B. y Gómez I., R. E. (1996). Proyectos Educativos Institucionales en Informática: Una estrategia sobre medida de formación de docentes de educación básica y media vocacional. ACUC: Noticias Informáticas, No 162, 32-34.

Maldonado G., L. F., y Andrade L., E. A. (1996). Ambiente Computarizado para el Aprendizaje Autodirigido del Diseño. Universidad Pedagógica Nacional. (En prensa).

Maldonado G., L. F. (1991a). Proyecto de Desarrollo en Tecnologías de la Información y la Comunicación para el Sector Educativo (TECNICE). Bogota: Universidad Pedagógica Nacional. Documento de circulación restringida.

Maldonado G., Luis F., Monroy H., L. B., Carrillo, Y. y Terreo G., A. (1978) Diseño y comparación de cuatro métodos de enseñanza en un curso introductorio de psicología del aprendizaje. Bogotá, D.C.: Universidad Pedagógica Nacional. 
McCulloch, W. S. and Pitts, W. (1943). A logical calculus of the ideas inmanent in nervous activity. Bulletin of Mathematical Biophisics, 5:115-137.

Mitchel, Melanie (1996). An introducción fo genétic algoníthms. Cambridge, Massachusetts: The MIT Press.

NELSON T. O., \& NARENS, L. (1990). Metamemory: A Theoretical Framework and New Findings. In G. Bower (De.). The Psychology of Learning and Motivation (Vol 26). New York: Academic Press.

Newell, A. and Simon, H. A. (1956). The logic theory machina. IRE Transactions no Information Theory. 2(3), 61-79.

Newell, A. and Simon, H. A. (1972). Human Problem Solving. Englewood Cliffts, NJ: Prentice Hall.

Newell, Allen (1981). The Knowledge Laval. Al Magazine, Summer, 1-20.

Osawa, E. (1995). A metalevel coordination strategy fon reactive cooperative planning. Proceedings of the First International Conference nn Murtiagent Systems ICMAS-95. Cambrige: AAAI Press/ The MIT Press, 65-72.

Papert. S. (1980). Mindstorms. New York: Basic Books.

Rich, E. y Knight. K. (1996). Inteligencia Artificial. Madrid McGraw Hill. Traducción del original. Artificial Intelligence.

Russell, S. y Norvig, P. (1996). Inteligencia Artificial Un en foque moderno. México: Prentice Hall. Traducido del Inglés: Artificial Intelligence a modern approach.

Samuel, A. L. (1959). Sorne studies in machina learning using the gama of checkers. IBM Journal of Research and Developmert, 3(3), 210-229.

Shannon, C. E. (1950). Programming a computar for playing chess. Philosophical Magazine. 4(4), 256-275.

Shannon. C. E. and Weaver, W. (1949). The mathematical theory of communication. Urbana The University of Illinois Press.

Shapiro, S. (1987), Editor. Encyclopedia of Artificial Intelligence. New York: John Willey \& Sons.

Simon, H. (1989). Prólogo a La Inteligencia Artificial y la Automática: aportación a la psicología del conocimiento. Barcelona: Editorial Herder. Traducción del Francés: Psycholgie, intelligence artificielle et automatique.

Singh, Jagjit (1966). Great ideas in information theory, language and cybernetics. Versión en español: Teoría de la información, del lenguaje y de la cibernética. Madrid: Alianza Universidad.

Sleeman, D. (1987). Micro SEARCH: a shell for building systems to help students solve non-deterministics tasks. En Kerasley, G.: Artificial intelligence \& instruction: 
applications and methods. Reading. Massachussetts: Addison-Wesley Publíshing Company.

Sowa, J. (1984). Conceptual Structures: ínformation processing in mmd and machina. Reading, Massachussetts: Addison-Wesley Publishing Company.

Steinberg, Ester (1992). The potential of Computer-Based Telecomunications for Instruction. Journal of Computar Basad Instruction, 19(2), 42-47.

Streitz, N. (1987). Mental modal and methaphors: implications for fha design of adaptive user-system interfaces. En Handl, $\mathrm{H}$. and Lesgold, A. Learning issues for intelligen tutoning systems. N.Y. : Springer-Verlag.

Sterling L. and Shapiro, E. (1986). The Art of Prolog: advance programming techniques. Cambridge, Massachussetts: The MIT Press.

Tanimoto, S. L. (1987). The elements of Artificial Intelligence: An introduction using LISP. Rockville, Maryland: Computer Science Press.

Thompson, P. (1987). Mathematical microworlds and intelligent computarassisted instruction. In kearsley, O. Editor: Artificial Intelligence and instruction: applications and methods. Reading, Massachussettes: Addison-Wesley Publishing Company.

Turing, A. M. (1936). On computable numbers, with an application fo the Entsheidungsproblem. Proceedings of the London Mathematical Society, 2nd series, 42: 230-265. Correction published in Vol 43: 544-546.

Turing, A. M. (1950). Computing machinery and intelligence. Mmd, october, 59, 433-460. In Feigenbaum and Feldman, Editors (1995): Computers \& Thought. Cambridge: AAAI Press / The MIT press.

Turing, A. M., Strachey, C. Bates, M. A. and Bowden, EV. (1953). Digital computers applied fo gamas. In Bowden, B V. editor: Faster than thought, pag. 286-316. London: Pitman.

Turkle, Sherry (1984). El segundo yo: los computadores y el espíritu humano. Traducción del original Inglés: The second salt: computers and. fha human spirit. Buenos Aires: Ediciones Galápago.

Vargas, G. (1986). Maleta Multimedial: derechos humanos, ética y moral. Santa Fe de Bogotá, D.C.: Universidad Pedagógica Nacional, Centro de Informática. Computen and system science, vol 133, 142-1 55.

Verdejo, MF. (1994). Collaborative Dialogue techniques in distance learning. Computer and system science, vol 133, 142-155.

Waterman, D. (1985). A guíde fo Expert Systems. Reading, Massachussetts. AddisonWesley Publishing Company.

Wenger, E. (1987). Artificial Intelligence and Tutoring Systems: computational and cognitive approaches to the communication of knowledge. Los Altos, CA.: Morgan Kaufmann Publishers, Inc. 
Winston, P. H. (1992). Artificial Inteligence (Third Edition). Reading, Massachussetts: Addison-Wesley Publishing. 